\title{
HI Supergiant Shells in the Large Magellanic Cloud
}

\author{
S. Kim ${ }^{1}$, L. Staveley-Smith ${ }^{2}$, R. J. Sault ${ }^{2}$, M. J. Kesteven ${ }^{2}$, \\ D. McConnell ${ }^{2}$, M. A. Dopita ${ }^{1}$ and M. Bessell ${ }^{1}$ \\ ${ }^{1}$ Mount Stromlo and Siding Spring Observatories, Weston Creek PO, ACT 2611, Australia \\ sek@mso.anu.edu.au \\ ${ }^{2}$ Australia Telescope National Facility, CSIRO, PO Box 76, Epping, NSW 2121, Australia
}

Received 1997 August 1, accepted 1997 December 30

\begin{abstract}
The recently completed HI mosaic survey of the Large Magellanic Cloud (Kim et al. 1997) reveals complex structure in the interstellar medium, including filaments, arcs, holes and shells. We have catalogued giant and supergiant HI shells and searched for correlations with $\mathrm{H} \alpha$ emission, using a new image taken with a camera lens mounted on the 16-inch telescope at Siding Spring Observatory.
\end{abstract}

Keywords: galaxies: Large Magellanic Cloud — interstellar: $\mathrm{HI}, \mathrm{H} \alpha-$ radio lines: atomic

\section{Introduction}

A rather complete review of recent work related to the Large Magellanic Cloud (LMC) has been given by Westerlund (1997). The overall distribution of the neutral hydrogen gas in the LMC and its relation to the SMC and the Galaxy had been described in the papers of McGee \& Milton (1966), Rohlfs et al. (1984) and Luks \& Rohlfs (1992). The neutral hydrogen (HI) gas in the LMC has been surveyed with the Australia Telescope Compact Array* (ATCA) at $\lambda 21 \mathrm{~cm}$. Compared to previous surveys of McGee \& Milton (1966), Rohlfs et al. (1984) and Luks \& Rohlfs (1992) using the Parkes telescope, the spatial resolution of the new survey is much higher: $1^{\prime}$ compared with $14^{\prime}-15^{\prime}$. We compare the large-scale distribution of $\mathrm{HI}$ in the LMC with the result of a recent $\mathrm{H} \alpha$ survey, taken with a camera lens mounted on the 16-inch telescope at Siding Spring Observatory. The $\mathrm{H} \alpha$ images cover the same area as the HI mosaic survey, and have a spatial resolution of $20^{\prime \prime}$, slightly better than the HI resolution. These two surveys combined offer a unique probe into the detailed relationship between the ionised and atomic phases in the interstellar medium of the LMC for the first time. The HI observations are summarised in Section 2, the $\mathrm{H} \alpha$ observations are summarised in Section 3 and results of correlations are given in Section 4 .

\section{HI Observations and Data Reduction}

The LMC was observed using four configurations of the ATCA in: 1994 October 26-November 9 (750D); 1995 February 23-March 11 (750A); 1995

\footnotetext{
* The Australia Telescope is funded by the Commonwealth of Australia for operation as a National Facility managed by CSIRO.
}

June 2-7 and 1995 October 15-31 (750B); and 1996 January 27-February 8 (750C). The combined configuration has 40 independent baselines ranging from 30 to $750 \mathrm{~m}$, with a baseline increment of $15 \cdot 3 \mathrm{~m}$. This results in an angular resolution of $1 .^{\prime} 0$ for the data presented here. The largest-scale structure that the present image is sensitive to is $\sim 0 .{ }^{\circ} 6$. The bandwidth used was $4 \mathrm{MHz}$, divided into 1024 channels (400 channels after the online application of Hanning smoothing and edge rejection). The observing band was centred on $1.419 \mathrm{GHz}$, corresponding to a central heliocentric velocity of $297 \mathrm{~km} \mathrm{~s}^{-1}$, a velocity coverage of -33 to $627 \mathrm{~km} \mathrm{~s}^{-1}$, and a velocity resolution of $1.65 \mathrm{~km} \mathrm{~s}^{-1}$.

The LMC was divided into 12 regions, each containing 112 pointing centres. Each pointing centre was observed for $15 \mathrm{~s}$, and good $u v$ coverage was obtained by returning to each pointing centre approximately 29 times during the course of a typical observing day of $15 \mathrm{~h}$. Each observation consisted of alternate scans of $28 \mathrm{~min}$ on the LMC and $3 \mathrm{~min}$ on the secondary (phase and amplitude) calibrator PKS B0407-658 or PKS B0454-810. The primary ATCA calibrator, PKS B1934-638 (assumed flux density $14.9 \mathrm{Jy}$ at $1.419 \mathrm{GHz}$ ), was observed at the start of each observing day. These observations served both for bandpass calibration and flux density calibration.

The data were edited, calibrated and mosaiced in MIRIAD. The pixel size is $20^{\prime \prime}$ and the size of the LMC cube is $1998 \times 2230 \times 120$. The $u v$ data were superuniformly weighted (Sramek \& Schwab 1989) with an additional Gaussian taper, Fouriertransformed and mosaiced in the image plane. The maximum entropy method was used to deconvolve this cube. The final cube was constructed by convolving the maximum entropy model with a 


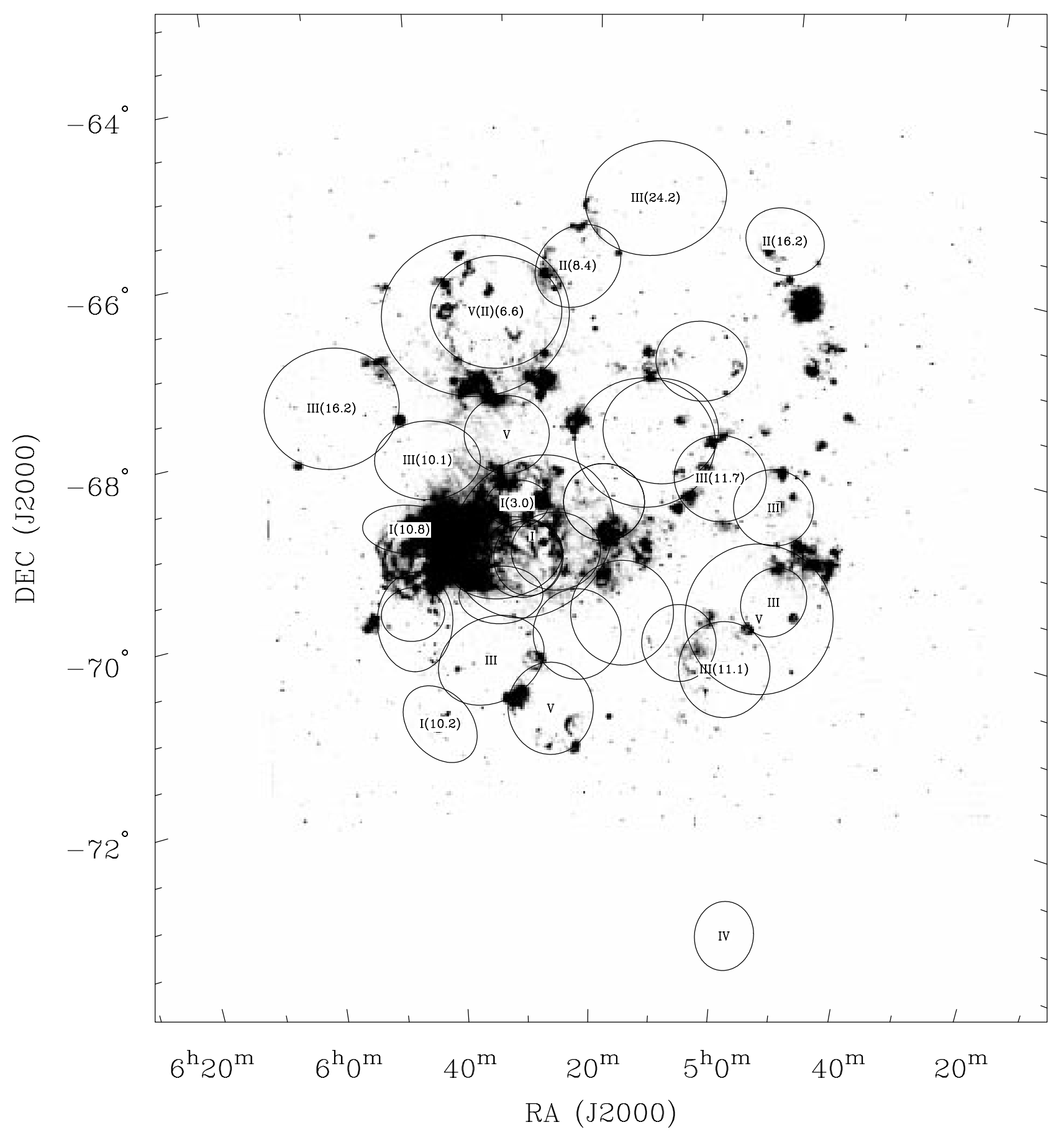

Figure 1-Positions of the candidate supergiant shells are overlayed as ellipses on the SSO H $\alpha$ image. The classification, based on a comparision between the HI and $\mathrm{H} \alpha$ properties, is marked (see text). The numbers indicate the dynamical ages of the shells in Myr.

Gaussian of FWHM $1^{\prime} \times 1^{\prime}$. The final rms noise, measured in line-free parts of the final cube, is $9 \mathrm{~K}$.

\section{H $\alpha$ Observations and Data Reduction}

For the wide-field survey of $\mathrm{H} \alpha$ emission in the LMC, a $153 \mathrm{~mm} \mathrm{f} / 5 \cdot 0$ camera lens from the $2 \cdot 3-\mathrm{m}$ telescope at Siding Spring Observatory was mounted on the 16-inch telescope with a filter assembly and a cooled $2048 \times 2048$ CCD. Each $15 \mu \mathrm{m}$ pixel corresponds to $20 .^{\prime \prime} 63$ on the sky, giving a total field size of $11 \cdot 7^{\circ}$ square. The $\mathrm{H} \alpha$ filter was centred at $6569 \AA$ with a FWHM of $15 \AA$. Continuum subtraction was done with another image centred at $6620 \AA$. The exposure time for both $\mathrm{H} \alpha$ and continuum images was $4 \times 900 \mathrm{~s}$.

\section{Results}

The peak brightness temperature map (Kim et al. 1997) of the LMC shows many filamentary features, combined with HI holes and shells. In our preliminary 


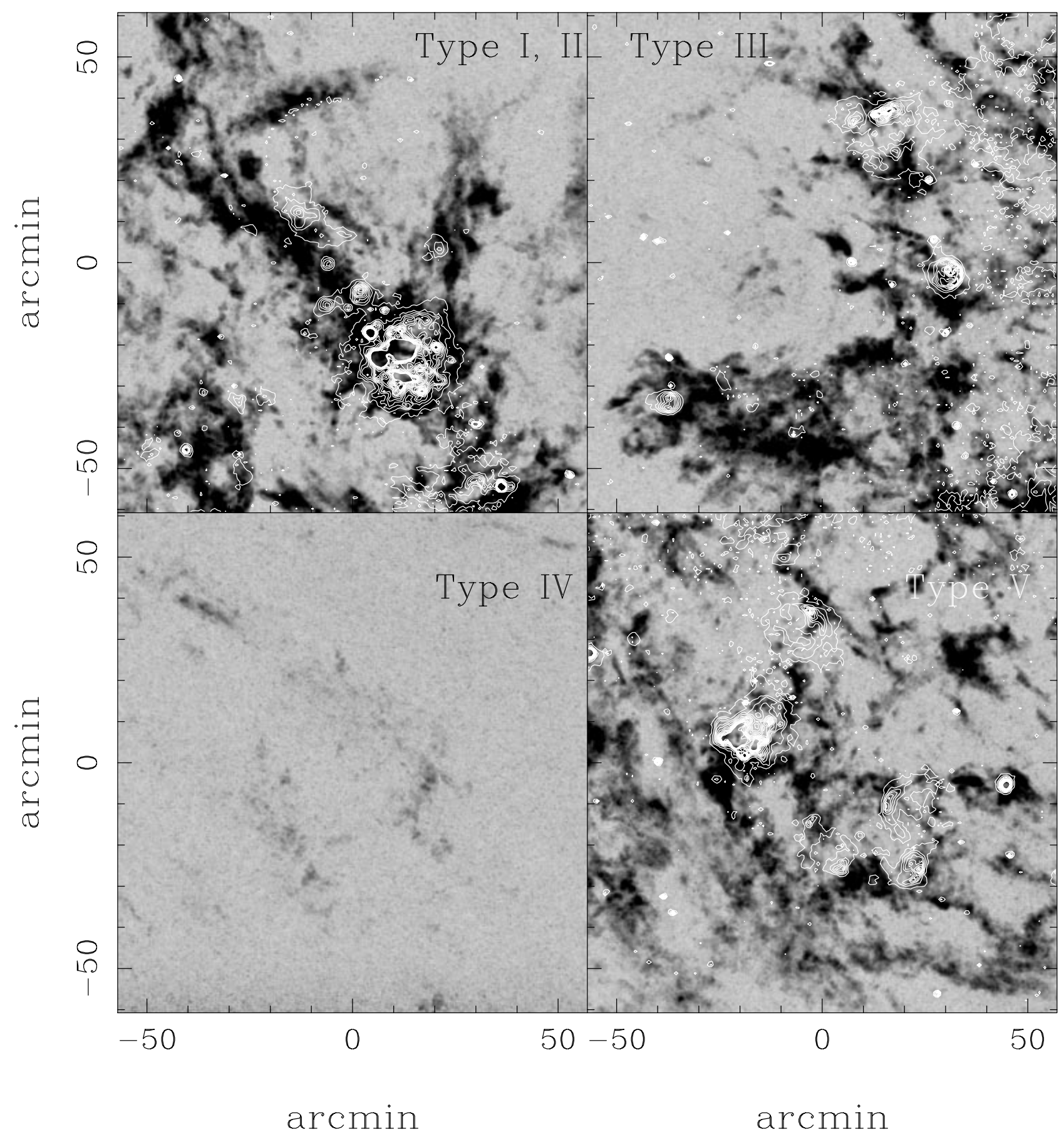

Figure 2-Geometrical relationship between HI (gray scale) and $\mathrm{H} \alpha$ (contours) for the five categories of HI shells described in the text.

analysis we have defined 32 HI supergiant shells; these shells are overlayed on the $\mathrm{H} \alpha$ image in Figure 1. The selection criteria applied to the shells are: (1) they must be edge-brightened ring-like structures larger than $600 \mathrm{pc}$ that are visible in at least three integrated channel maps (the spacing between each integrated channel map is $5 \mathrm{~km} \mathrm{~s}^{-1}$ ); and (2) the approaching and receding hemispheres (or one of the hemispheres) must be visible in position-velocity cuts ( $\mathrm{P}-\mathrm{V}$ diagrams).
Many of the shells are not simple expanding shells in the $\mathrm{P}-\mathrm{V}$ diagrams. The cause of this is a combination of confusion between neighbouring shells, lack of zero-spacing data and breakouts from the disk of the LMC. If we compare the distribution of HI supergiant shells in each of the four quadrants of the LMC, we clearly see that the largest number of shells is located in the south-eastern quadrant, near the 30 Doradus nebula, as seen in Figure 1. Figure 1 also shows the age of each shell, $t_{s}$ (in 
units of Myr), calculated from $t_{s}=\frac{3}{5} R_{s} / V_{s}$ as given by the standard theory of wind-driven bubbles (Weaver et al. 1977), where $R_{s}$ is the shell radius and $V_{s}$ is the expansion velocity of the shell. The measured ages of the expanding supergiant shells are distributed between 3 and 25 Myr. The shells in Figure 1 may also be classified morphologically by comparing their $\mathrm{HI}$ and $\mathrm{H} \alpha$ structures. We define five categories for the geometrical correlation between $\mathrm{HI}$ and $\mathrm{H} \alpha$ emission (Figure 2). These categories should approximate an age sequence. In Type I shells, the HI shells are filled with ionised gas or else include discrete HII regions inside the HI shell. In Type II shells, the ionised gas forms a thin shell and has been trapped by the HI shell. Type III represents HI shells that have discrete HII regions on the wall of the shell. Type IV represents HI shells that are not associated with ionised gas. Type III and Type IV may both have evolved from Type II, with the difference between the two types probably dependent on the density of the interstellar medium. Type V are HI shells that have morphologically complex structure, which might be due to distortions caused by the secondary star formation on the surface of the shell. Age estimates for some of the shells marked in Figure 1 are due to uncertain or unmeasurable expansion velocities.

Kim, S., Staveley-Smith, L., Sault, R. J., McConnell, D., Kesteven, M. J., \& Freeman, K. C. 1997, PASA, 14, 119 Luks, T., \& Rohlfs, K. 1992, A\&A, 263, 41

McGee, R. X., \& Milton, J. A. 1966, Aust. J. Phys., 19, 343

Rohlfs, K., Kreitschmann, J., Siegman, B. C., \& Feitzinger, J. V. 1984, A\&A, 137, 343

Sramek, R. A., \& Schwab, F. R. 1989, in Synthesis Imaging in Radio Astronomy, ed. R. A. Perley et al. (San Francisco: ASP), p. 123

Weaver, R., McCray, R., Castor, J., Shapiro, P., \& Moore, R. 1977, ApJ, 218, 377

Westerlund, B. E. 1997, The Magellanic Clouds (Cambridge Univ. Press) 